UPR-886-T

\title{
Chiral Models of Weak Scale Supersymmetry
}

\author{
Jens Erler \\ Department of Physics and Astronomy, University of Pennsylvania, \\ Philadelphia, PA 19104-6396 \\ E-mail:erler@langacker.hep.upenn.edu
}

\begin{abstract}
I discuss supersymmetric extensions of the Standard Model containing an extra $U(1)^{\prime}$ gauge symmetry which provide a solution to the $\mu$-problem and at the same time protect the proton from decaying via dimension 4 operators. Moreover, all fields are protected by chirality and supersymmetry from acquiring high scale masses. The additional requirements of anomaly cancellation and gauge coupling unification imply the existence of extra matter multiplets and that several fields participate in $U(1)^{\prime}$ symmetry breaking simultaneously. While there are several studies addressing subsets of these requirements, this work uncovers simultaneous solutions to all of them. It is surprising and encouraging that extending the Minimal Supersymmetric Standard Model by a simple $U(1)$ factor solves its major drawbacks with respect to the non-supersymmetric Standard Model, especially as current precision data seem to offer a hint to the existence of its corresponding $Z^{\prime}$ boson. It is also remarkable that there are many solutions where the $U(1)^{\prime}$ charges of the known quarks and leptons are predicted to be identical to those $E_{6}$ motivated $Z^{\prime}$ bosons which give the best fit to the data. I discuss the solutions to these constraints including some phenomenological implications.
\end{abstract}

\section{Introduction}

Besides its conceptual elegance, the prime motivation to consider low energy supersymmetry (SUSY) [1] is the stabilization of the electroweak scale under radiative corrections. However, the Minimal Supersymmetric Standard Model (MSSM) provides no explanation to the $\mu$-problem [2], i.e., to why the scales of SUSY breaking and of the supersymmetric bilinear Higgs $\mu$-term,

$$
\mu h_{d} h_{u}
$$

are of comparable magnitude. The MSSM should therefore be extended by at least some sector solving the $\mu$-problem. In this sense, the term MSSM is a misnomer.

Moreover, in sharp contrast to the non-supersymmetric Standard Model (SM) where the stability of the proton can be understood entirely by gauge invariance, the MSSM predicts rapid proton decay via dimension 4 operators, unless an additional discrete symmetry, such as $R$ parity, is imposed ad hoc. While this is a logical possibility, it severely compromises the initial elegance and one prefers to save the successful features of the SM when exploring its alternatives. 
It has also been argued [3] that those discrete symmetries' which survive as remnants of some underlying string model, might either be unsuited to forbid (at least) the dangerous dimension 4 operators, or arise only along some fine-tuned flat scalar field direction. Indeed, baryon number conservation by the principles of gauge invariance (and renormalizability) has been on the wish list of SUSY practitioners for almost two decades [5].

It is well known that either of the problems addressed in the previous two paragraphs can be solved by the introduction of an extra $U(1)^{\prime}$ symmetry. Examples for either case are reviewed in the next section using $U(1)$ groups motivated by $E_{6}$ Grand Unified Theories (GUTs). Additional $U(1)$ s have long been considered as very well motivated extensions of the MSSM [6]; they are predicted in most GUTs and appear copiously in superstring theories. In this article, I address the question whether it is possible to find models in which an extra $U(1)^{\prime}$ solves both problems simultaneously.

If such an extension is free of gauge and gravitational anomalies, one can allow a grand desert scenario between the electroweak scale and a more fundamental high mass scale. This is desirable since our confidence in the MSSM is boosted by the observation of approximate gauge coupling unification [7] (as predicted by the simplest and most economic GUT and string models) at a scale somewhat below the Planck scale. An extra $U(1)^{\prime}$ does not affect the renormalization group equations at the one-loop level, and it is conceivable that the quality of unification even improves at two-loop precision. In order to rigorously protect the electroweak scale from higher mass scales, I also demand that the field content of the models be completely chiral before gauge and supersymmetry breaking. I.e., all superfields should transform nontrivially with respect to the low-energy $S U(3) \times S U(2) \times U(1)_{Y} \times U(1)^{\prime}$ gauge symmetry, and all bilinear (mass) terms must be forbidden by gauge invariance. Note, that these requirements present a strong set of constraints, and it is not clear a priori, whether any solutions exist. The key ingredient necessary to arrive at a solution is that at least two MSSM singlet fields which are charged under the $U(1)^{\prime}$ have to acquire vacuum expectation values (VEVs).

Electroweak precision data imply further hints and constraints. The predictions of the SM are generally in very good agreement with experiments provided the Higgs boson mass, $M_{H}$, is smaller than about $250 \mathrm{GeV}$ [8,9]. This is also predicted by the MSSM and many extensions $\left(M_{H} \lesssim 150 \mathrm{GeV}\right)$ [11]. Moreover, the superpartners and extra Higgs fields decouple from the precision observables over large parts of the MSSM parameter space, so that the MSSM is likewise in agreement with observations [12]. Addition of an extra $Z^{\prime}$ boson can even improve the global fit to all data [13], driven mostly by the parity violation experiments in Cs [14, 115], but the results in $\mathrm{Tl}$ [16], and from the $Z$ lineshape measurements at LEP 1 [17] also play a role. Note, that these fits suggest a $Z^{\prime}$ boson mass $M_{Z^{\prime}} \lesssim \mathcal{O}(1 \mathrm{TeV})$ and continue to yield results on $M_{H}$ consistent with SUSY [13].

Fits to the oblique parameters, $S, T$, and $U$ [18] (or $\epsilon_{1}, \epsilon_{2}$, and $\epsilon_{3}$ [19]), describing new physics contributions to vector boson self-energies, yield results consistent with zero [9] (for Higgs masses in the MSSM range). This implies restrictions on extra matter fields which can contribute significantly to $S$ and $T$, but if they are non-chiral (with respect to the SM gauge group) and approximately mass degenerate their effects are rendered small. If they arise in

\footnotetext{
${ }^{1}$ Continuous global symmetries are not expected to arise from string theory [4].
} 
complete representations of $S U(5)$, they do not affect gauge coupling unification at the oneloop level, nor its scale. However, in the bottom-up approach of this paper I do not assume an $S U(5)$-GUT symmetry. I will show that other matter configurations can preserve unification, as well.

To reiterate, I will assume that there is an additional $U(1)^{\prime}$ symmetry with a mechanism built in to solve the SUSY $\mu$-problem. I will classify the solutions to the conditions of anomaly cancellation, gauge coupling unification, and chirality. To avoid potential problems with fractionally charged states, I will also assume $S U(5)$-type charge quantization. About half of the resulting models predict a sufficiently long proton lifetime.

In the next section, I review the situation for the most popular $U(1)^{\prime}$ extensions arising from $S O(10)$ and $E_{6}$ GUTs [20]. In Section 3, I introduce the anomaly conditions and show that it is not possible to find chiral models if only one MSSM singlet field develops a VEV. Section 4 shows that there are 33 solutions (family universal with respect to the ordinary fermions) if there are two singlets acquiring VEVs in the course of $U(1)^{\prime}$ breaking. Five of these models involve a number of $\mathbf{5}+\overline{\mathbf{5}}$ representations and singlets of $S U(5)$, but no extra matter beyond that. Allowing incomplete representations of $S U(5)$ and the weaker requirements of gauge unification and absence of fractionally charged states yield the remaining 28 solutions. I will also briefly address the prospects to derive these models from the $E_{8} \times E_{8}$ heterotic string theory. Some of the phenomenological implications of the type of models obtained in Section 1 are discussed in Section 5. My conclusions are presented in Section 6.

\section{$2 \quad E_{6}$ inspired $U(1)^{\prime}$ models}

Consider, as an illustrative example, the case of an $E_{6}$ gauge group which is free of anomalies in all its representations. A fundamental 27 representation contains a pair of $S U(2)$ doublets, $h_{d}$ and $h_{u}$, carrying the appropriate $U(1)_{Y}$ hypercharges to serve as the MSSM Higgs doublets, i.e., allowing the Yukawa couplings,

$$
q h_{d} \bar{d}, \quad q h_{u} \bar{u}, \quad l h_{d} e^{+}, \quad l h_{u} \bar{\nu} .
$$

Here $l$ and $q$ are the lepton and quark doublet superfields 2 , $e^{+}, \bar{d}$, and $\bar{u}$ denote the $S U(2)$ singlets, and $\bar{\nu}$ refers to the right-handed neutrino superfield. If one identifies the $U(1)^{\prime}$ with the $U(1)_{\psi}$ defined by $E_{6} \rightarrow S O(10) \times U(1)_{\psi}$, then $h_{d}$ and $h_{u}$ have equal $U(1)^{\prime}$ charges, excluding an elementary $\mu$-term. A 27 also includes an MSSM singlet superfield, $S$, which has the right $U(1)^{\prime}$ charge to allow a trilinear Yukawa term,

$$
S h_{d} h_{u}
$$

in the superpotential. If the scalar component of $S$ develops a VEV triggered by SUSY breaking, it breaks the $U(1)^{\prime}$ gauge symmetry and induces an effective $\mu$-term, linking these scales. Since it is assumed that electroweak symmetry breaking is triggered by SUSY breaking, as well, all scales are related and the $\mu$-problem is solved [21,22]. Moreover, one predicts $M_{Z^{\prime}} \lesssim \mathcal{O}(1 \mathrm{TeV})$

\footnotetext{
${ }^{2}$ I denote the MSSM multiplets by lower case letters, while additional fields will be capitalized.
} 
if one wants to avoid excessive fine-tuning. The $U(1)_{\psi}$ symmetry also forbids all baryon number, $\mathbf{B}$, and lepton number, $\mathbf{L}$, violating terms in the renormalizable superpotential of the MSSM,

$$
l h_{u}, \quad l l e^{+}, \quad l q \bar{d}, \quad \overline{d d} \bar{u} .
$$

However, each 27 also contains an extra pair of $S U(2)$ singlet quarks, $D$ and $\bar{D}$. Upon $U(1)^{\prime}$ breaking they are expected to receive $\mathrm{TeV}$ scale masses through terms of the form?

$S D \bar{D}$

but they also allow new $\mathbf{B}$ and $\mathbf{L}$ violating (though $\mathbf{B}-\mathbf{L}$ conserving) terms,

$$
q q D, \quad \bar{d} \bar{D} \bar{u} .
$$

Together with the $\mathbf{B}$ and $\mathbf{L}$ conserving operators,

$$
l_{q} \bar{D}, \quad e^{+} D \bar{u}, \quad \bar{\nu} D \bar{d},
$$

dimension 4 proton decay is reintroduced through the exchange of $D$ and $\bar{D}$ quarks.

Clearly, one can avoid this problem by restricting oneself to $S O(10)$ and identifiying the $U(1)^{\prime}$ with the $U(1)_{\chi}$ appearing in $S O(10) \rightarrow S U(5) \times U(1)_{\chi}$, which likewise forbids the terms in Eq. (田). The ordinary fermions and the $\bar{\nu}$ complete anomaly free $\mathbf{1 6}$ representations (avoiding the extra singlet quarks), and one has to add a pair of Higgs doublets which by anomaly cancellation are required to have equal and opposite $U(1)^{\prime}$ charges. As a result, a primordial $\mu$-term is allowed, and any term of the form (3) can only include a $U(1)^{\prime}$ neutral singlet field, $S$. Therefore, both aspects of the $U(1)^{\prime}$ solution to the $\mu$-problem (exclusion of an elementary $\mu$ term and generation of an effective $\mu$-term at the $\mathrm{TeV}$ scale) are lost, while there is no problem with too rapid proton decay. This is the reversed situation compared to the $U(1)_{\psi}$ case.

One possibility is to ignore the $\mu$-problem. Models containing an anomaly free $U(1)^{\prime}$ symmetry designed to stabilize the proton have been discussed by various authors [3,5,23]. Conversely, non-anomalous $U(1)$ 's addressing the $\mu$-problem without reference to proton decay are discussed in Ref. [24]. An interesting solution to both problems is to flip the signs of the hypercharge assignments of the $D$ and $\bar{D}$ quarks relative to the $E_{6}$ case [25]. This does not alter the SM anomaly conditions, and forbids the dangerous terms in Eq. (6). However, it implies the existence of stable fractionally charged baryons with TeV scale masses. Such states have not been observed, yet, and lead to serious cosmological problems. If the reheating temperature after inflation is of the order of the mass of these exotic particles or above, their hadronic interactions give rise to rather large number densities of these stable hadrons [26], orders of magnitude above experimental bounds [27]. Lowering the reheating temperature does not solve the problem either, since successful baryogenesis probably requires a reheating temperature after inflation at least of the order of the critical temperature of the electroweak phase transition of about $100 \mathrm{GeV}$ [28]. Then a significant number density of $\mathrm{TeV}$ scale particles can still be

\footnotetext{
${ }^{3}$ Terms of this type can also trigger radiative $U(1)^{\prime}$ symmetry breaking in analogy to electroweak symmetry breaking in the MSSM, provided the coupling strength is comparable to the top quark Yukawa coupling [22].

${ }^{4}$ I am indebted to Michael Plümacher for discussions on this point.
} 


\begin{tabular}{|cccc|cccc|}
\hline$\left(\begin{array}{c}\nu \\
e^{-}\end{array}\right)$ & -1 & & & $\begin{array}{c}\bar{\nu} \\
e^{+}\end{array}$ & $+5 / 3$ & $+\tilde{a}$ & $+2 \tilde{b}$ \\
+ & $-\tilde{a}$ & $+\tilde{b}$ \\
\hline$\left(\begin{array}{c}u \\
d\end{array}\right)$ & $+1 / 3$ & & $+\tilde{b}$ & $\begin{array}{c}\bar{u} \\
\bar{d}\end{array}$ & $\begin{array}{c}+1 / 3 \\
-1\end{array}$ & $\begin{array}{c}+\tilde{a} \\
-\tilde{a}\end{array}$ & $+\tilde{b}$ \\
\hline \hline$\left(\begin{array}{c}N \\
E^{-}\end{array}\right)$ & $+2 / 3$ & $+\tilde{a}$ & $-\tilde{b}$ & $\frac{D}{D}$ & $\begin{array}{c}-2 / 3 \\
+2 / 3\end{array}$ & $\begin{array}{r}-2 \tilde{b} \\
-\tilde{b}\end{array}$ \\
\hline$\left(\begin{array}{c}E^{+} \\
\bar{N}\end{array}\right)$ & $-2 / 3$ & $-\tilde{a}$ & $-2 \tilde{b}$ & $S$ & 0 & & $+3 \tilde{b}$ \\
\hline
\end{tabular}

Table 1: $U(1)^{\prime}$ charges of a $\mathbf{2 7}$ representation for an arbitrary $E_{6}$ boson [13. The upper half of the Table with $\tilde{b}=0$ corresponds to a family in $S O(10)$ GUT. The doublets on the left-hand side are the fields $l, q, h_{d}$, and $h_{u}$, respectively. The special cases $(\tilde{a}, \tilde{b})=(0,0)$, $(0,-4 / 3)$, and $(-5 / 3,0)$ correspond, respectively, to the $U(1)_{\chi}$, the $U(1)_{\psi}$, and the $U(1)_{Y}$ (up to normalization).

produced by the high-energy tail of thermal particle distributions, or during reheating itself [29]. The present work is therefore devoted to models without fractionally charged states, i.e., $S U(5)$ charge quantization is imposed,

$$
Q+\frac{\mathcal{C}_{3}}{3}=Q_{Y}+\frac{\mathcal{C}_{2}}{2}+\frac{\mathcal{C}_{3}}{3} \in \mathbb{Z},
$$

where $Q$ is electric charge, $Q_{Y}$ is hypercharge, and where $\mathcal{C}_{2}$ is an even (odd) integer for vector (spinor) representations of $S U(2)$. Similarly, $\mathcal{C}_{3}$ is the triality class of $S U(3)$ (e.g. \pm 1 for quarks and antiquarks).

All of the $E_{6}$ inspired models discussed above have another unwanted feature in the context of gauge coupling unification, which requires the addition of an extra pair of $S U(2)$ doublets. By virtue of anomaly cancellation these must be non-chiral and one would therefore need a mechanism preventing them from receiving high scale tree-level masses. This is not a problem in the context of string models which predict the absence of fundamental mass parameters in the low energy effective Lagrangian by conformal invariance. However, as these models stand, the extra doublets would then be massless. One could couple them to an overall singlet receiving a $\mathrm{VEV}$, but in general this is not protected to be of electroweak size. Alternatively, one can assume that the extra doublets develop VEVs, as well, but it seems difficult to arrange that all chargino and neutralino masses are compatible with the experimental lower mass limits as some masses would be predicted to be strictly of order the $Z$ boson mass, $\lesssim M_{Z}$. This paper is therefore devoted to stabilize electroweak scale physics by the principles of gauge and supersymmetry, while assuring that all fields receive large enough masses after symmetry breaking.

An alternative way to remove the dangerous terms in Eq. (6) would be to choose a particular linear combination of $U(1)_{\chi}, U(1)_{\psi}$, and $U(1)_{Y}$. Table 1 shows that the condition,

$$
\tilde{a}+2 \tilde{b}+\frac{5}{3}=0,
$$


allows a Dirac mass term, $D \bar{d}$, so that $D$ and $\bar{d}$ can be removed from the spectrum. Baryon number is now conserved because the trilinear terms in Eq. (4) are still forbidden, and effects from the ones in Eq. (6) decouple. The role of $\bar{d}$ is now played by $\bar{D}$, but since $l q \bar{D}$ is the only type of operator involving it, $l$ must act as a Higgs superfield, and trade its role with $h_{d}$. However, Eq. (9) also implies that $l$ and $h_{u}$ have equal and opposite $U(1)^{\prime}$ charges and furthermore allows $\bar{\nu}$ to decouple from the spectrum. Hence, we recover the $S O(10)$ case (with $\bar{\nu}$ replaced by $S$ ) and the $\mu$-problem which comes with it. Eq. (9) defines therefore an alternative set of $S O(10)$ models within $E_{6}$, in analogy with the alternative left-right model [30].

\section{Anomaly cancellation in the presence of an extra $U(1)^{\prime}$}

Consider the $U(1)^{\prime}$ charge assignment in Table 2. Indicated are three generations of ordinary matter fields, one pair of Higgs doublets, $h_{d}$ and $h_{u}$, appropriate to break electroweak symmetry, one singlet Higgs, $S$, for $U(1)^{\prime}$ symmetry breaking, and further singlet fields?, $T_{i}$, which are (initially) assumed not to receive VEVs. Shown are also examples of extra matter fields, where $E^{-}$are charged lepton singlets, $L$ are lepton doublets, $U$ and $D$ are up-type and down-type quark singlets, $Q$ are quark doublets, and where $E^{+}, \bar{L}, \bar{U}, \bar{D}$, and $\bar{Q}$ are their mirror partners. $W$ and $G$ are neutral fields under the $U(1)_{Y}$ transforming in the adjoint representations of $S U(2)$ and $S U(3)$, respectively. These are the fields which will play a role in the models surviving the analysis in Section 4 , but initially any field respecting Eq. (8) is allowed. I assume family universality for the ordinary fermions and also the exotic fields, i.e., the new fields have identical $U(1)^{\prime}$ charges in the cases of multiplicities greater than one, but this assumption is not crucial and can be relaxed easily.

As usual, all ordinary charged leptons and quarks are expected to become massive through the Yukawa couplings $[$ in Eq. (2). On the other hand, the extra fields, such as $D$ and $E$, are assumed to get vector-like (with respect to the SM group) masses induced by trilinear couplings with $S$ and their respective $S U(5)$ mirror partners, as for example in Eq. (5). This way their contributions to oblique parameters decouple. If an irreducible representation is real, $\mathbf{r}=\overline{\mathbf{r}}$, and has $Q^{\prime}=-s / 2$, it can acquire a supersymmetric Majorana mass through a trilinear coupling to $S$. Examples are the fields $W$ and $G$, and in this case it suffices to add a single copy. Finally, I demand,

$$
s=-\left(c_{1}+c_{2}\right) \neq 0,
$$

to guarantee a solution to both aspects of the $\mu$-problem.

Since the exotic matter is vector-like with respect to the SM gauge group it does not spoil the anomaly cancellation present there. Triangle diagrams with two SM (i.e., either $S U(3)$, or $S U(2)$, or $\left.U(1)_{Y}\right)$ and one $U(1)^{\prime}$ gauge fields as external legs yield three conditions which have to be satisfied. At first sight one would expect that there are many solutions given the many free parameters in Table 2, but as it will turn out there are none. For example, if the exotic matter consists of $k_{5}$ copies of $\mathbf{5}+\overline{\mathbf{5}}$ representations of $S U(5)$, the mixed $S U(3) / U(1)^{\prime}$ anomaly

\footnotetext{
${ }^{5}$ I will refer to a singlet as a right-handed neutrino, $\bar{\nu}$, if it admits the Yukawa coupling in Eq. (2).

${ }^{6}$ While $m_{u}=0$ is strongly disfavored, it is not firmly ruled out. I will comment on this case later in this Section.
} 


\begin{tabular}{|c|c|c||c|c|c|}
\hline & $Q_{Y}$ & $Q^{\prime}$ & & $Q_{Y}$ & $Q^{\prime}$ \\
\hline \hline$\left(\begin{array}{c}\nu \\
e^{-}\end{array}\right)$ & $-1 / 2$ & $a$ & $\bar{\nu}$ & 0 & $-\left(a+c_{2}\right)$ \\
$\left(\begin{array}{c}u \\
d\end{array}\right)$ & $+1 / 6$ & $b$ & $\bar{u}$ & $-2 / 3$ & $-\left(b+c_{1}\right)$ \\
& & $e^{+}$ & $+1 / 3$ & $-\left(b+c_{1}\right)$ \\
\hline$h_{d}$ & $-1 / 2$ & $c_{1}$ & $h_{u}$ & $+1 / 2$ & $c_{2}$ \\
\hline \hline$D$ & $-1 / 3$ & $d_{1}$ & $\bar{D}$ & $+1 / 3$ & $-\left(s+d_{1}\right)$ \\
$L$ & $-1 / 2$ & $d_{2}$ & $\bar{L}$ & $+1 / 2$ & $-\left(s+d_{2}\right)$ \\
$Q$ & $+1 / 6$ & $d_{3}$ & $\bar{Q}$ & $-1 / 6$ & $-\left(s+d_{3}\right)$ \\
$U$ & $+2 / 3$ & $d_{4}$ & $\bar{U}$ & $-2 / 3$ & $-\left(s+d_{4}\right)$ \\
$E^{-}$ & -1 & $d_{5}$ & $E^{+}$ & +1 & $-\left(s+d_{5}\right)$ \\
$W$ & 0 & $-s / 2$ & $G$ & 0 & $-s / 2$ \\
\hline$S$ & 0 & $s$ & $T_{i}$ & 0 & $t_{i}$ \\
\hline
\end{tabular}

Table 2: $U(1)_{Y}$ and $U(1)^{\prime}$ charge assignments for the MSSM matter fields including righthanded neutrinos and the two Higgs doublets (upper half), a number of singlets $S$ and $T_{i}$, and extra exotic fields. Three generations of ordinary fermions are assumed and generation indices suppressed. Table 1 corresponds to $a=-1$ (normalization), $c_{1}+c_{2}=-s=-(a+3 b)$, and $d_{1}=-2 b$.

condition reads,

$$
\left(k_{5}-3\right)\left(c_{1}+c_{2}\right)=0,
$$

and together with Eq. (10) fixes $k_{5}=3$. Eq. (11) is independent of the $d_{i}$, and so are the mixed $S U(2) / U(1)^{\prime}$ and $U(1)_{Y}^{2} / U(1)^{\prime}$ conditions, which are, respectively,

$$
\begin{aligned}
\left(k_{5}+1\right)\left(c_{1}+c_{2}\right)+3(a+3 b) & =0, \\
\left(\frac{10}{3} k_{5}-14\right)\left(c_{1}+c_{2}\right)-6(a+3 b) & =0 .
\end{aligned}
$$

This is solved only by the $S O(10)$-type relation, $a=-3 b$, and for equal and opposite Higgs charges, $c_{1}=-c_{2}$.

This conclusion cannot be avoided even if we generalize to arbitrary extra matter fields of the form $\mathbf{N}+\overline{\mathbf{N}}$, where $\mathbf{N}$ is any (in general reducible) representation of $S U(5)$. Eqs. (11)-(13) still apply, but $k_{5}$ is now defined as the index of $\mathbf{N}$,

$$
k_{5}=2 \sum_{r} \frac{n_{r} \operatorname{dim} \mathbf{r}}{\operatorname{dim} S U(5)} C_{r}
$$

where the sum is over the irreducible representations, $\mathbf{r} \in \mathbf{N}$. $n_{r}$ are the multiplicities $\left(n_{r}=1 / 2\right.$ for Majorana types) and $C_{r}$ is the second-order Casimir invariant of representation $\mathbf{r}$. One can also allow extra matter fields in incomplete representations of $S U(5)$. However, preservation of gauge coupling unification enforces the differences of the one-loop $\beta$-function coefficients to be the same as in the MSSM[? This leaves only one adjustable parameter, the index $k_{5}$, and the anomaly conditions remain unchanged.

\footnotetext{
${ }^{7}$ I will refer to such configurations as quasi-complete $S U(5)$ representations.
} 
The conclusion, $c_{1}+c_{2}=0$, is also independent of the assumption of family universality. If, for example, the $U(1)^{\prime}$ charges of the third generation were different from the two lighter ones and associated with parameters $a^{\prime}$ and $b^{\prime}$ (cf. Table 2), then this would merely result in the replacement,

$$
3(a+3 b) \longrightarrow 2 a+a^{\prime}+3\left(2 b+b^{\prime}\right)
$$

in Eqs. (12) and (13).

A more interesting case [31] can be constructed from the one in the previous paragraph by replacing $-\left(b+c_{2}\right)$ by $-\left(b^{\prime}+c_{2}\right)$ for the quark singlets $\bar{u}$ and $\bar{c}$, and conversely for $\bar{t}$. This results in a zero eigenvalue for the up-type quark mass matrix, and would either require $m_{u}=0$, or that a non-vanishing $m_{u}$ can be generated radiatively, non-perturbatively, or by some other mechanism. Family non-universal $U(1)^{\prime}$ charges can also induce significant flavor changing neutral current effects [32]. If one allows for this possibility, Eqs. (11)-(13) are replaced by,

$$
\left(\begin{array}{ccc}
0 & +1 & k_{5}-3 \\
+1 & -3 & k_{5}+1 \\
-2 & \frac{34}{3} & \frac{10}{3} k_{5}-14
\end{array}\right)\left(\begin{array}{c}
2 a+a^{\prime}+9 b \\
b-b^{\prime} \\
c_{1}+c_{2}
\end{array}\right)=0 .
$$

However, the determinant of this matrix is non-vanishing $(=-4)$ independently of $k_{5}$, and there is only the trivial solution implying $b=b^{\prime}$, i.e., nothing new.

The setup discussed in this section does not seem to provide solutions to the $\mu$-problem. It should be stressed, however, that this conclusion depends crucially on the additional assumptions of gauge coupling unification and chirality. For example, the model in Ref. 25] is chiral, but fails to produce unification; alternatively, if unification is enforced by adding an extra pair of doublets it turns non-chiral. Another example can be constructed with the assignments in Table 2. Suppose the exotic matter consists of the fields $(Q+\bar{Q})+(U+\bar{U})$. Eq. (11) is then satisfied $\left(k_{5}=3\right)$, and Eq. (13) becomes equivalent to Eq. (12), which are solved by, $4\left(c_{1}+c_{2}\right)=-3(a+3 b)$. The quadratic anomaly condition from triangle graphs with one hypercharge and two $U(1)^{\prime}$ gauge bosons in the external legs depends on $d_{3}$ and $d_{4}$ and can easily be solved. Extra singlets, $T_{i}$, can be added to satisfy the cubic (pure $U(1)^{\prime}$ ) and trace (mixed gravitational $\left./ U(1)^{\prime}\right)$ anomaly conditions. This model is chiral but the gauge couplings do not unify. Unification can be arranged by adding an $E^{ \pm}$pair to complete a $\mathbf{1 0}+\overline{\mathbf{1 0}}$ representation of $S U(5)$, but it would have to carry equal and opposite $U(1)^{\prime}$ charges, spoiling the chirality of the model. Thus, this model mirrors the situation in Ref. 25 except that it has no fractionally charged baryons.

\section{Two Higgs singlet solutions}

Suppose now that besides $S$ another MSSM singlet field, $T=T_{0}$, with $Q^{\prime}=t=t_{0}$, develops a VEV. A subset of the exotic matter fields, $\mathbf{M}+\overline{\mathbf{M}} \subset \mathbf{N}+\overline{\mathbf{N}}$, could then acquire TeV scale masses through trilinear couplings to $T$, provided the $U(1)^{\prime}$ charge assignments of the fields in $\mathbf{M}$ and $\overline{\mathbf{M}}$ are changed 8 from $d_{i}$ and $-\left(s+d_{i}\right)$ to $e_{i}$ and $-\left(t+e_{i}\right)$, respectively. The anomaly

\footnotetext{
${ }^{8}$ In general, this explicitly violates the assumption of universality w.r.t. the exotic fields which was made in Section $\beta$.
} 
conditions in Eqs. (11)-(13) now change to,

$$
\left(\begin{array}{ccc}
0 & k_{5}-k_{3}-3 & k_{3} \\
-1 & k_{5}-k_{2}+1 & k_{2} \\
+2 & \frac{10}{3}\left(k_{5}-k_{1}\right)-14 & \frac{10}{3} k_{1}
\end{array}\right)\left(\begin{array}{c}
3(a+3 b) \\
s \\
t
\end{array}\right)=0
$$

where $k_{3}$ is the $S U(3)$ index of $\mathbf{M}, k_{3} \operatorname{dim} S U(3)=2 \sum_{r} m_{r} \operatorname{dim} \mathbf{r} C_{r}$. Similarly, $k_{2}$ is the $S U(2)$ index of $\mathbf{M}$, and $k_{1}=6 / 5 \operatorname{Tr}_{\mathbf{M}} Q_{Y}^{2}$. The $S U(5)$ charge quantization condition (8) implies,

$$
\frac{5}{6} k_{1}-\frac{1}{2} k_{2}-\frac{1}{3} k_{3} \equiv k_{0} \in \mathbb{Z}
$$

Notice, that $k_{3}, k_{2}$, and $k_{0}$ are integers, and that by definition, $0 \leq k_{j} \leq k_{5}$ for $1 \leq j \leq 3$, which implies the bound $\left|k_{0}\right| \leq 5 k_{5} / 6$. If the determinant of the matrix in Eq. (17) is non-vanishing, there is only the unwanted solution, implying $c_{1}+c_{2}=0$. The matrix becomes singular for,

$$
k_{3}=\left(k_{5}-3\right)\left(k_{0}+k_{2}-k_{3}\right)
$$

Using the definitions,

$$
\sigma \equiv \frac{(a+3 b)}{s}, \quad \tau \equiv \frac{t}{s}
$$

the solutions can be classified as follows:

- The case $k_{3}=k_{0}+k_{2}=0$ implies $5 k_{1}=-3 k_{2}$, and therefore $k_{0}=k_{1}=k_{2}=\sigma=\tau=0$ independently of $k_{5}$, which will be rejected because it corresponds essentially to the nonchiral situation in Section 3. This is the only possibility for $k_{5}=1$.

- If $k_{5} \geq 5$, Eq. (19) implies that $k_{3}$ is an integer multiple of $k_{5}-3$, and $k_{j} \leq k_{5}$ requires $k_{3}=k_{5}-3$ and $k_{0}+k_{2}=k_{5}-2$. One has $3 \sigma=\left(k_{5}-k_{2}+1\right)$ and $\tau=0$, i.e., these models are also non-chiral since the fields within the $\mathbf{M}+\overline{\mathbf{M}}$ representation are not tied to $U(1)^{\prime}$ symmetry breaking. Such solutions exist for $k_{5}=3$ (e.g., the three generation $E_{6}$ model with an extra pair of doublets) and $k_{5}=4$, as well, but they will be discarded.

- $k_{5}=2$ implies $k_{0}+k_{2}=0, k_{3}>k_{2}, 3 \sigma=3+k_{2} / k_{3}$ and $\tau=1+1 / k_{3}$. There are three solutions, but the case $\left(k_{3}, k_{2}, k_{1}, k_{0}\right)=(2,1,1 / 5,-1)$ cannot be made consistent with the $S U(5)$ quantization condition.

- $k_{5}=3$ implies $k_{3}=0,3 \sigma=3+k_{0} /\left(k_{0}+k_{2}\right)$ and $\tau=1-1 /\left(k_{0}+k_{2}\right)$. There are eight solutions of this type.

- $k_{5}=4$ implies $k_{3}=2, k_{0}+k_{2}=4, \sigma=5 / 3-k_{2} / 6$ and $\tau=1 / 2$. There are two solutions, but the case $\left(k_{3}, k_{2}, k_{1}, k_{0}\right)=(2,3,19 / 5,1)$ cannot be made consistent with the $S U(5)$ quantization condition.

Thus, there are an infinite number of non-chiral solutions, but only 11 chiral ones, which are collected in Table 3. All of them predict $\tau=t / s \geq 0$, implying that there are no $D$-flat scalar field directions. Furthermore, all of them predict $k_{5} \leq 4$, which guarantees that the 


\begin{tabular}{|c|c|c|c|c|c|c|c|c|c|c|}
\hline solution & $k_{5}$ & $k_{3}$ & $k_{2}$ & $k_{1}$ & $k_{0}$ & $\sigma$ & $\tau$ & Yukawa couplings to $T$ & $\omega_{1}$ & $\omega_{3}^{\max }$ \\
\hline I & 2 & 1 & 0 & $2 / 5$ & 0 & 1 & 2 & $(D+\bar{D})$ & 0 & $-3 / 2$ \\
\hline II & 2 & 2 & 0 & $4 / 5$ & 0 & 1 & $3 / 2$ & $2(D+\bar{D})$ & $1 / 2$ & $23 / 16$ \\
\hline III & 3 & 0 & 2 & $6 / 5$ & 0 & 1 & $1 / 2$ & $2(L+\bar{L})$ & $3 / 2$ & $3 / 2$ \\
\hline IV & 3 & 0 & 3 & $9 / 5$ & 0 & 1 & $2 / 3$ & $3(L+\bar{L})$ & $4 / 3$ & $31 / 27$ \\
\hline $\mathrm{V}$ & 4 & 2 & 4 & $16 / 5$ & 0 & 1 & $1 / 2$ & all but $2(D+\bar{D})$ & $3 / 2$ & $9 / 16$ \\
\hline$E_{6}$ & 3 & 0 & 1 & $3 / 5$ & 0 & 1 & 0 & $(L+\bar{L})$ & 2 & 2 \\
\hline $\mathrm{VI}$ & 3 & 0 & 3 & $3 / 5$ & -1 & $5 / 6$ & $1 / 2$ & $W+(L+L)$ & & \\
\hline VII & 3 & 0 & 1 & $9 / 5$ & 1 & $7 / 6$ & $1 / 2$ & $(L+\bar{L})+E^{ \pm}$ & & \\
\hline VIII & 3 & 0 & 2 & $12 / 5$ & 1 & $10 / 9$ & $2 / 3$ & $2(L+\bar{L})+E^{ \pm}$ & & \\
\hline IX & 3 & 0 & 3 & 3 & 1 & $13 / 12$ & $3 / 4$ & $3(L+\bar{L})+E^{ \pm}$ & & \\
\hline $\mathrm{X}$ & 3 & 0 & 0 & $12 / 5$ & 2 & $4 / 3$ & $1 / 2$ & $2 E^{ \pm}$ & & \\
\hline $\mathrm{XI}$ & 3 & 0 & 1 & 3 & 2 & $11 / 9$ & $2 / 3$ & $(L+\bar{L})+2 E^{ \pm}$ & & \\
\hline
\end{tabular}

Table 3: Classification of solutions to the conditions of anomaly cancellation, chirality, $S U(5)$ charge quantization, and gauge coupling unification. It is assumed that two fields participate in $U(1)^{\prime}$ gauge symmetry breaking. For comparison, the non-chiral three generation $E_{6}$ solution with an extra neutral $(t=0)$ Higgs singlet giving mass to an extra vector-like pair of doublets $\left(k_{2}=1\right)$ is also shown. $\omega_{1}$ and $\omega_{3}$ are shown for $\mathbf{5}+\overline{5}$ representations only, in which case they are defined in Eqs. (21) and (25), respectively. The column before these specifies the fields receiving masses through trilinear couplings to $T$ (cf. Table 4 below). In addition, $2(L+\bar{L})$ fields can be replaced by the combination $W+E^{ \pm}$, where applicable.

gauge couplings remain perturbative up to the unification scale, while $k_{5}=5$ would yield non-perturbative unification [33].

In general, each solution in Table 3 can be realized with various exotic matter contents. Observe that the configurations of $k_{i}$ in the Table restrict the possible matter content to the fields displayed in Table 2. These fields can be combined into quasi-complete $S U(5)$ representations shown in Table 4 .

In the remainder of this Section and in the next Section I will focus on exotic matter in complete $\mathbf{5}+\overline{\mathbf{5}}$ representations of $S U(5)$, unless noted otherwise. In this case one can write the sum over the other singlet charges, which is fixed by the trace condition, $\operatorname{Tr} Q^{\prime}=0$, as

$$
\omega_{1} \equiv \frac{1}{s} \sum_{i=1} t_{i}=5 k_{5}+(\tau-1)\left(3 k_{3}+2 k_{2}-1\right)-12 .
$$

Interestingly, when 3 right-handed neutrinos are included, $\omega_{1}$ is completely determined within each model. For $\mathbf{5}+\overline{\mathbf{5}}$ representations one has $k_{0}=0$, and then Eqs. (10), (17), and (18) imply the relation,

$$
s=-\left(c_{1}+c_{2}\right)=(a+3 b),
$$

which is familiar from the $E_{6}$ assignment in Table 1. Thus, most of the phenomenological studies of $E_{6}$ inspired $Z^{\prime}$ bosons apply here, and also to the other solutions with $k_{0}=0$. To simplify the algebra, I impose the orthogonality condition, $\operatorname{Tr} Q_{Y} Q^{\prime}=0$, which can be relaxed at a later 


\begin{tabular}{|c|c|c|c|c|c|c|c|c|c|}
\hline representation & $k_{5}$ & $D+\bar{D}$ & $L+\bar{L}$ & $Q+\bar{Q}$ & $U+\bar{U}$ & $E^{ \pm}$ & $W$ & $G$ & solution(s) \\
\hline 1 & 2 & 1 & - & - & 1 & - & 1 & - & $\mathrm{I}$ \\
2 & 2 & 2 & - & - & - & 1 & 1 & - & I, II \\
3 & 2 & 2 & 2 & - & - & - & - & - & $\mathrm{I}, \mathrm{II}$ \\
\hline 4 & 3 & - & 1 & - & - & 2 & 1 & 1 & III, IV, VI-XI \\
5 & 3 & - & 3 & - & - & 1 & - & 1 & III, IV, VII-IX \\
6 & 3 & 1 & - & 1 & - & 2 & - & - & $\mathrm{X}$ \\
7 & 3 & 2 & 1 & - & 1 & - & 1 & - & $\mathrm{VI}$ \\
8 & 3 & 3 & 1 & - & - & 1 & 1 & - & III, IV, VI, VII \\
9 & 3 & 3 & 3 & - & - & - & - & - & $\mathrm{III}, \mathrm{IV}$ \\
\hline 10 & 4 & 2 & - & - & 2 & - & 2 & - & $\mathrm{V}$ \\
11 & 4 & 2 & 1 & 1 & - & 2 & - & - & $\mathrm{V}$ \\
12 & 4 & 3 & - & - & 1 & 1 & 2 & - & $\mathrm{V}$ \\
13 & 4 & 3 & 2 & - & 1 & - & 1 & - & $\mathrm{V}$ \\
14 & 4 & 4 & - & - & - & 2 & 2 & - & $\mathrm{V}$ \\
15 & 4 & 4 & 2 & - & - & 1 & 1 & - & $\mathrm{V}$ \\
16 & 4 & 4 & 4 & - & - & - & - & - & $\mathrm{V}$ \\
\hline
\end{tabular}

Table 4: Quasi-complete $S U(5)$ representations (preserving gauge coupling unification) which can be used to realize the solutions in Table 3. There are other configurations with $k_{5}=4$, but solution $\mathrm{V}$ requires at least two pairs of $D+\bar{D}$ quarks. Similarly, the $\mathbf{1 0}+\overline{\mathbf{1 0}}$ representation of $S U(5)\left(k_{5}=3\right)$ cannot be used for any of the solutions in Table 3 and has been omitted.

stage. Combined with the anomaly condition quadratic in the $U(1)^{\prime}$ charges? $\operatorname{Tr} Q_{Y} Q^{\prime 2}=0, \mathrm{I}$ find,

$$
\begin{aligned}
2\left(k_{3} e_{1}+k_{2} e_{2}\right) & =9 \frac{(a-b)+\left(c_{1}-c_{2}\right)}{\tau-1}-\tau\left(k_{3}+k_{2}\right) s \\
2\left[\left(k_{3}-k_{5}\right) d_{1}+\left(k_{2}-k_{5}\right) d_{2}\right] & =\frac{(6 \tau+3)(a-b)+(7 \tau+2)\left(c_{1}-c_{2}\right)}{\tau-1}+\left[2 k_{5}-\left(k_{3}+k_{2}\right)\right] s .
\end{aligned}
$$

Similarly, the cubic condition, $\operatorname{Tr} Q^{\prime 3}=0$, reads,

$$
\begin{gathered}
\omega_{3} \equiv \frac{1}{s^{3}} \sum_{i=1} t_{i}^{3}=5 k_{5}+\left(\tau^{3}-1\right)\left(3 k_{3}+2 k_{2}-1\right)-\frac{3}{4}\left[3+9\left(\frac{a-b}{s}\right)^{2}+4\left(\frac{c_{1}-c_{2}}{s}\right)^{2}\right]+\quad(25) \\
3\left[3 \tau k_{3} \frac{e_{1}}{s}\left(\frac{e_{1}}{s}+\tau\right)+2 \tau k_{2} \frac{e_{2}}{s}\left(\frac{e_{2}}{s}+\tau\right)+3\left(k_{5}-k_{3}\right) \frac{d_{1}}{s}\left(\frac{d_{1}}{s}+1\right)+2\left(k_{5}-k_{2}\right) \frac{d_{2}}{s}\left(\frac{d_{2}}{s}+1\right)\right],
\end{gathered}
$$

and one can show that,

$$
\omega_{3} \leq \frac{1}{4}\left[5\left(k_{5}-1\right)+\left(\tau^{3}-1\right)\left(3 k_{3}+2 k_{2}\right)\right]-\tau^{3} .
$$

\footnotetext{
${ }^{9} \operatorname{Tr} Q_{Y} Q^{\prime 2}$ also vanishes if $s=0$.
} 
The inequality (26) is saturated when,

$$
a=b=\frac{s}{4}, \quad c_{1}=c_{2}=d_{1}=d_{2}=-\frac{s}{2}, \quad e_{1}=e_{2}=-\frac{\tau}{2} s,
$$

which yields a class of $Z^{\prime}$ bosons which couple like the $Z_{\psi}$ to the ordinary fermions and the Higgs doublets. This is encouraging as the recent analysis [13] of precision data revealed an excellent fit to the $Z_{\psi}$ assuming some amount of mixing with hypercharge. Recall that $U(1)^{\prime}$ subgroups from $E_{6}$ are frequently discussed because they represent manifestly anomaly free examples (and because they arise in popular GUT and string models), but the $E_{6}$ gauge symmetry and Yukawa coupling relations are usually explicitly broken to avoid serious conflicts with observation (such as the proton lifetime). Here we recover $U(1)^{\prime} \equiv U(1)_{\tilde{\psi}}$ symmetries which are very similar to the $U(1)_{\psi}$ from a very different bottom-up approach.

Relaxing the orthogonality condition, $\operatorname{Tr} Q_{Y} Q^{\prime}=0$, and using the trivial solution to Eq. (17), $a+3 b=c_{1}+c_{2}=s=t=0$, one can construct another family of special solutions, $U(1)_{\chi}$, where the MSSM fields have charges as in the $U(1)_{\chi}$ case. While they would yield non-chiral pairs of Higgs doublets, these solutions could be interesting if they enter linear combinations with the $U(1)_{\tilde{\psi}}$ or if the corresponding $Z_{\tilde{\chi}}$ coexists with the $Z_{\tilde{\psi}}$. The quadratic anomaly condition now reduces to

$$
\left(k_{3}-k_{5}\right) d_{1}+\left(k_{2}-k_{5}\right) d_{2}-\tau\left(k_{3} e_{1}+k_{2} e_{2}\right)+2\left(a+c_{1}\right)=0,
$$

where solutions with $c_{1}=a$ correspond to hypercharge and $c_{1}=-2 a / 3$ to the $U(1)_{\tilde{\chi}}$. Note, that there are other solutions to the anomaly constraints with $s=0$, but only those satisfying Eq. (28) can mix with the chiral $U(1)^{\prime}$ s.

The encounter of the $U(1)_{\tilde{\psi}}$ and $U(1)_{\tilde{\chi}}$ groups is also encouraging from a top-down perspective. There are classes of string models where gauge coupling unification is predicted without the appearance of a full GUT group in the zero-slope limit. Examples are heterotic string constructions where the gauge group factors considered are realized at the same KacMoody level, $k$, or open string models with the group factors arising from the same $D$-brane. The $E_{8} \times E_{8}$ heterotic string is particularly suited here, because it offers the prerequisite $E_{6}$ subgroups. The reference $E_{8}$ symmetry implies the relevant matching (unification) conditions, as well as quantization conditions for its $U(1)$ subgroups. The latter are given by

$$
Q_{\chi}-\frac{6}{5} Q_{Y} \in \mathbb{Z}, \quad Q_{\psi}-\frac{5}{4} Q_{\chi} \in \mathbb{Z}, \quad Q_{\phi}-\frac{4}{3} Q_{\psi} \in \mathbb{Z},
$$

where the last relation refers to the $U(1)_{\phi}$ defined by $E_{7} \rightarrow E_{6} \times U(1)_{\phi}$. The normalizations are chosen such that $Q_{Y}=1$ for $e^{+}(a=-1 / 2), Q_{\chi}=1$ for $\bar{\nu}(a=-3 / 5), Q_{\psi}=1$ for $S(s=1)$, and $Q_{\phi}=1$ for one of the $E_{6}$ singlets within the 56 of $E_{7}$. Eq. (28) implies for the $U(1)_{\tilde{\chi}}$ of solution II, $2 d_{2}+3 e_{1}+2 / 5=0$, or when combined with the first Eq. (29), $e_{1}+2 / 5 \in 2 \mathbb{Z}$. With the exception of two fields, the second Eq. (29) is also satisfied. But the $D$ quarks have the sign of their $U(1)_{\tilde{\psi}}$ charges reversed and the $T$ field $\left(Q_{\tilde{\chi}}=0\right)$ has half-integer instead of integer $U(1)_{\tilde{\psi}}$ charge. It is not clear at present四 whether $E_{8}$ charge quantization, i.e., Eqs. (29), are

\footnotetext{
${ }^{10}$ In Kac-Moody level 1 models $S U(5)$ charge quantization can never be obtained unless a complete $S U(5)$ GUT group survives 34. On the other hand in the level 2 models or Ref. 35] the third Eq. (29) is respected by all fields.
} 
predicted in string models with gauge coupling unification and $S U(5)$ charge quantization. If so one would have to impose the condition $\tau \in \mathbb{Z}$, and only solution I would survive.

The $E_{8}$ embedding also allows one to fix the overall normalization (coupling strength) of the $U(1)^{\prime}$. In units in which an $E_{8}$ root vector $P$ has length $P^{2}=1$, one has $P^{2}=3 / 5$ for a 10 of $S U(5), P^{2}=5 / 8$ for a $\mathbf{1 6}$ of $S O(10), P^{2}=2 / 3$ for a $\mathbf{2 7}$ of $E_{6}$, and $P^{2}=3 / 4$ for a 56 of $E_{7}$. The 10 of $S U(5)$ is relevant here, because it contains the $S U(3) \times S U(2)$ singlet with unit charge (the $e^{+}$) and can therefore serve to properly normalize the hypercharge gauge coupling, $g_{Y}$, in terms of one of the non-Abelian gauge couplings, $g$. Assuming the normalization in

Eq. (29) one finds the well-known result, $g_{Y}=\sqrt{3 / 5} g$. Notice, that this argument needs no reference to a complete GUT group like $S U(5)$, but it applies only to string constructions in which the embedding into $E_{8}$ is still traceable. Referring again to the normalization fixed by Eq. (29) one finds in a similar way, $g_{\chi}=\sqrt{5 / 8} g$ and $g_{\psi}=\sqrt{2 / 3} g$.

The reference $E_{8}$ root vector also allows to set lower limits on the possible Kac-Moody levels, $k$. For example, the singlet $T$ of solution II has $Q_{\tilde{\psi}}=3 s / 2$, which implies $P_{T}^{2}=3 / 2>1$ and means that $T$ cannot be obtained in the massless sector if $k=1$, while $k \geq 2$ would be allowed by this consideration. Interestingly, the theorem about fractionally charged states in level $k=1$ models [34] implies the same conclusion.

\section{$5 \quad$ Phenomenological aspects}

The one-loop $\beta$-function coefficient for the $U(1)^{\prime}$ is given by,

$$
\begin{gathered}
\frac{\beta_{1}^{\prime}}{s^{2}}=\omega_{2}+5 k_{5}+\left(\tau^{2}-1\right)\left(3 k_{3}+2 k_{2}+1\right)+6+9\left(\frac{a-b}{s}\right)^{2}+7\left(\frac{c_{1}-c_{2}}{s}\right)^{2}+ \\
2\left[3 k_{3} \frac{e_{1}}{s}\left(\frac{e_{1}}{s}+\tau\right)+2 k_{2} \frac{e_{2}}{s}\left(\frac{e_{2}}{s}+\tau\right)+3\left(k_{5}-k_{3}\right) \frac{d_{1}}{s}\left(\frac{d_{1}}{s}+1\right)+2\left(k_{5}-k_{2}\right) \frac{d_{2}}{s}\left(\frac{d_{2}}{s}+1\right)\right],
\end{gathered}
$$

where $\omega_{2}=s^{-2} \sum_{t=i} t_{i}^{2}$. $\beta_{1}^{\prime}$ is minimized when Eqs. (27) are satisfied, i.e., for the $U(1)_{\tilde{\psi}}$, in which case one finds,

$$
\beta_{1}^{\prime}=\frac{1}{3}\left[5\left(k_{5}+2\right)+\left(\tau^{2}-1\right)\left(3 k_{3}+2 k_{2}\right)+2\left(\tau^{2}+\omega_{2}\right)\right] .
$$

In Eq. (31) I have chosen the $E_{6}$ inspired normalization, $s^{2}=2 / 3$. For comparison, the $S U(3) \times S U(2) \times U(1)_{Y}$ one-loop $\beta$-function coefficients are $\beta_{3}=k_{5}-3, \beta_{2}=k_{5}+1$, and $\beta_{1}=k_{5}+33 / 5$ (using $S U(5)$ normalization, $a^{2}=3 / 20$ ), respectively.

For concreteness, I now focus on the $Z_{\tilde{\psi}}$ of solution II which is reminiscent of an $E_{6}$ model with three families of $\mathbf{2 7}$, but with one copy of $D+\bar{D}$ quarks removed and the $U(1)^{\prime}$ charges of the other two copies altered. The trace and cubic anomaly conditions, $\omega_{1}=1 / 2$ and $\omega_{3}=\omega_{3}^{\max }=23 / 16$, can be satisfied by adding, for example, $S U(5)$ singlets with $U(1)^{\prime}$ charges, $t_{1}=t_{2}=s, t_{3}=3 s / 4, t_{2 i+2}=-s / 2$, and $t_{2 i+3}=s / 4$, where $1 \leq i \leq 9$, or in shorthand notation, $t_{i} \sim\left[1^{2}, 3 / 4,-(1 / 2)^{9},(1 / 4)^{9}\right]$. There are no singlets with charges -1 or $-3 / 2$ 


\begin{tabular}{|c|c|c||c|c|c|}
\hline & $Q_{Y}$ & $Q_{\tilde{\psi}}^{(\mathrm{II})}$ & & $Q_{Y}$ & $Q_{\tilde{\psi}}^{(\mathrm{II})}$ \\
\hline \hline$\left(\begin{array}{c}\nu \\
e^{-}\end{array}\right)$ & $-1 / 2$ & $+1 / 4$ & $\bar{\nu}$ & 0 & $+1 / 4$ \\
$\left(\begin{array}{c}u \\
d\end{array}\right)$ & $+1 / 6$ & $+1 / 4$ & $\bar{u}$ & $-2 / 3$ & $+1 / 4$ \\
& & & $\bar{d}$ & $+1 / 3$ & $+1 / 4$ \\
\hline$h_{d}$ & $-1 / 2$ & $-1 / 2$ & $h_{u}$ & $+1 / 2$ & $-1 / 2$ \\
\hline \hline$D_{1,2}$ & $-1 / 3$ & $-3 / 4$ & $\bar{D}_{1,2}$ & $+1 / 3$ & $-3 / 4$ \\
$L_{1,2}$ & $-1 / 2$ & $-1 / 2$ & $\bar{L}_{1,2}$ & $+1 / 2$ & $-1 / 2$ \\
$S$ & 0 & +1 & $T$ & 0 & $+3 / 2$ \\
$T_{1,2}^{+1}$ & 0 & +1 & $T_{3}^{+3 / 4}$ & 0 & $+3 / 4$ \\
$T_{2 i+2}^{-1 / 2}$ & 0 & $-1 / 2$ & $T_{2 i+3}^{+1 / 4}$ & 0 & $+1 / 4$ \\
\hline
\end{tabular}

Table 5: Charge assignment for the $Z_{\tilde{\psi}}$ of solution II realized with a $\mathbf{5}+\overline{\mathbf{5}}$ of $S U(5)$. The $U(1)^{\prime}$ normalization corresponds to $s=1$.

in this choice, assuring that the crucial fields, $S$ and $T$, cannot acquire high scale masses. The quantum numbers for this case, $Z_{\tilde{\psi}}^{(\mathrm{II})}$, are summarized in Table 5 .

There are a total of 12 fields with quantum numbers like the right-handed neutrinos. Since these fields are protected from acquiring high scale Majorana masses, one predicts a Dirac mass matrix with three electroweak scale eigenvalues. I.e., there are unacceptably large neutrino masses, unless the Yukawa couplings are chosen zero or tiny. A clean way to cure this problem is to add the set of singlets $\square, t_{i} \sim 3\left[1,-(3 / 4)^{4},(1 / 2)^{6},-(1 / 4)^{4}\right]$. Now the fields with $t_{i}= \pm s / 4$ (and some others) can form bilinear Dirac mass terms, resulting in a neutrino mass matrix with three zero eigenvalues, which correspond predominantly to the left-handed neutrinos provided the bilinear masses are much larger than the Yukawa mass terms. Clearly, the bilinear masses can be taken to infinity and the corresponding fields can be smoothly removed from the spectrum, yielding the residual chiral set of singlets (now including $S, T$, and the three $\bar{\nu}), \sim\left[3 / 2,1^{6},-(3 / 4)^{11},(1 / 2)^{9}\right]$.

There is another interesting solution which can be obtained by adding the fields, $t_{i} \sim$ $3\left[-(5 / 4), 1^{4},-(3 / 4)^{6},(1 / 2)^{4},-(1 / 4)\right]$ to the ones in Table 5. This results in the chiral singlet set, $\sim\left[3 / 2,-(5 / 4)^{3}, 1^{15},-(3 / 4)^{17},(1 / 2)^{3},(1 / 4)^{9}\right]$, where the fields with $Q^{\prime}=-5 s / 4$ allow trilinear couplings with $S$ and the $\bar{\nu}$. This once again results in three zero eigenvalues, and if the masses generated by $S$ are an order of magnitude larger than the ones from the standard Yukawa terms, these also correspond predominantly to the left-handed neutrinos, but the mixing with the massive states could induce a small amount of missing invisible $Z$ width, $\Gamma_{\text {inv }}$. Indeed, the LEP Collaborations [17] currently report a shortage of about $0.5 \%$ in $\Gamma_{\text {inv }}$. Furthermore, the Fermi constant, $G_{F}$, extracted from $\mu$ decays could increase, significantly increasing the extracted Higgs boson mass from electroweak fits (the central value of which is currently below the lower search limit [9]) but potentially deteriorating the quality of the global fit to

\footnotetext{
${ }^{11}$ By virtue of the identity, $n^{3}-4(n-1)^{3}+6(n-2)^{3}-4(n-3)^{3}+(n-4)^{3}=0$, such a configuration does not alter $\omega_{1}$ or $\omega_{3}$.
} 
all data. There can be other effects associated with this scenario, such as a violation of weak charged current universality. There are strong limits on the non-universality between the first two families, however, driven by the well measured branching ratio, $\frac{\mathcal{B}\left(\pi \rightarrow \mu \nu_{\mu}\right)}{\mathcal{B}\left(\pi \rightarrow e \nu_{e}\right)}$. Thus the effect in $\Gamma_{\text {inv }}$ should be expected to be dominated by the $\nu_{\tau}$ sector, in agreement with the experience that the third family Yukawa couplings are typically the largest.

Yet another solution to the problem of neutrino mass would be to implement the see-saw mechanism which can be achieved by choosing a linear combination of the $U(1)_{\tilde{\psi}}$ and the $U(1)_{\tilde{\chi}}$, such that the $\bar{\nu}$ are $U(1)^{\prime}$ neutral and allowed to receive high scale Majorana masses. As is well known, this also produces small but non-vanishing Majorana masses for the left-handed neutrinos, which is not the case for the scenarios in the previous two paragraphs. However, one should generally expect non-vanishing masses for the left-handed neutrinos originating from non-renormalizable terms suppressed by powers of the high mass scale [36]. Dimension 5 operators would yield the same kind of suppression as in the see-saw mechanism, but higher order suppressions (by intermediate scales) are conceivable ${ }^{\text {[2] }}$.

As for baryon number violating interactions, the $U(1)_{\tilde{\psi}}$ symmetry of solution II forbids the terms in Eq. (6), thus assuring proton stability at the renormalizable level. Dimension 5 operators can also lead to proton decay at rates conflicting with the experimental limits. However, the $D$-terms,

$$
q q \bar{d}^{*}, \quad q q \bar{D}^{*}, \quad D D \bar{u}^{*}, \quad \bar{u} \bar{d} D^{*}, \quad \bar{u} \bar{D} D^{*},
$$

are also absent by virtue of the $U(1)_{\tilde{\psi}}$, and likewise for the $F$-terms,

$$
l q q q, \quad H_{d} q q q, \quad L q q q, \quad H_{u} q D D, \quad \bar{L} q D D, \quad e^{+} D D D . \quad e^{+} \bar{u} \bar{d} .
$$

On the other hand, the $F$-terms,

$$
\bar{\nu} q q D, \quad \overline{\nu u} \overline{d D}, \quad T_{i}^{-3 / 4} \bar{u} \overline{d d}, \quad T_{i}^{+5 / 4} \bar{u} \overline{D D},
$$

are guaranteed to be absent only when there are no MSSM singlets with $Q^{\prime}=5 s / 4, s / 4$, or $-3 s / 4$. While this can be arranged, there is another dimension $5 F$-term,

$$
e^{+} \overline{u u} \bar{D}
$$

which is similar to the operator $e^{+} \bar{u} \bar{d}$, present in the $R$-parity conserving version of the MSSM. There one has to assume that its corresponding coupling strength is small, and taking into account suppressions from first generation Yukawa couplings, Cabibbo mixing, and phase space, the resulting proton lifetime can be acceptable. In comparison, the term (35) is less concerning, because at the renormalizable level the $D$ quarks couple only (ignoring the possibility of a $T_{i}^{+1 / 2} \bar{d} D$-term) via gauge interactions and the $T D \bar{D}$-term, both conserving " $D$-number". Therefore, observable proton decay is not expected, unless a large $\overline{d D}$ mixing effect can be generated. Alternatively, the critical dimension 5 F-term in Eq. (35) can be removed if there is an admixture of the $U(1)_{\tilde{\chi}}$. Thus, choosing $k_{3}=k_{5}$ (or more generally coupling all of the exotic quarks to singlets other than $S$ ) is an efficient strategy to achieve a realistic proton lifetime.

\footnotetext{
${ }^{12}$ Employing the $Z^{\prime}$ scale, $M_{Z^{\prime}} \approx 800 \mathrm{GeV}$ 13], and the unification scale, $M \sim 2 \times 10^{16} \mathrm{GeV}$, suggests neutrino masses of order $M_{Z^{\prime}}^{2} / M \sim 0.03 \mathrm{eV}$, in excellent agreement with the mass scale inferred from atmospheric neutrino oscillations 37]. One might wonder whether this agreement not only signals the presence of a new physics scale close to the unification scale, but possibly even suggests the absence of further scales below that.
} 


\section{Conclusions}

I have presented a class of models with an additional non-anomalous $U(1)^{\prime}$ in which gauge couplings unify, the $\mu$-problem can be solved, and electric charge is quantized. These models are chiral, protecting a large desert between the electroweak (SUSY breaking) and unification (string) scales. Moreover, in some of these models the $U(1)^{\prime}$ is sufficient to avoid conflicts with experimental bounds on the proton lifetime. Within a deliberately traditional and conservative framework the analysis presented is rather general, but there are various directions which can be chosen to go beyond it.

For example, I assumed that two MSSM singlet fields participate in $U(1)^{\prime}$ symmetry breaking. Only a finite number of solutions exist since the anomaly matrix in Eq. (17) has to be singular. The analysis generalizes straightforwardly if one allows three (or more) singlets to acquire VEVs. In this case an infinite number of solutions exist, a large (but finite) number of which being consistent with perturbative gauge coupling unification. A preliminary study of this class reveals that unlike the solutions in Table 3 there are also models with $D$-flat scalar field directions, a property which can be phenomenologically advantageous. There are also solutions simultaneously in accordance with $E_{8}$ charge quantization (see Eq. (29)) and the proton lifetime.

I have further assumed that all exotic fields receive their masses through couplings to MSSM singlets acquiring VEVs upon $U(1)^{\prime}$ breaking. But exotic leptons participating in $S U(2) \times U(1)_{Y}$ symmetry breaking will in general receive mass contributions of $\mathcal{O}\left(M_{Z}\right)$ which could conceivably by themselves be large enough to be in agreement with current limits. This seems difficult but is a possible loophole which I have ignored in this work.

An implicit assumption has been made concerning the precise form of gauge coupling unification. It is the often made assertion that gauge coupling unification works satisfactorily in the MSSM and should not be altered in leading (one-loop) order. Extra matter multiplets are then usually restricted to appear exclusively in complete $S U(5)$ representations. In the present work, I have already relaxed this restriction and included quasi-complete SU(5) representations. However, gauge coupling unification works only approximately, with the prediction for the strong coupling constant, $\alpha_{s}\left(M_{Z}\right)$, significantly higher than most observations and the unification scale at least one order of magnitude lower than the string scale. It is fair to ask whether some arbitrary (incomplete from the perspective of $S U(5)$ ) representation could improve the quality of unification. Indeed, by including a set of fields which contributes stronger to the $S U(3) \beta$-function than to the one of $S U(2)$ improves the prediction for $\alpha_{s}\left(M_{Z}\right)$. Furthermore, by having the smallest contribution to the $U(1)_{Y} \beta$-function, one can increase the unification scale. The minimal choice is the set $G+W+E^{ \pm}$, contributing $\Delta \beta_{3}=3, \Delta \beta_{2}=2$, and $\Delta \beta_{1}=6 / 5$, respectively. Alternatively, one could add another $\mathbf{5}+\overline{\mathbf{5}}$ of $S U(5)$ to this set, or choose a family plus a mirror-family of quarks, $Q+\bar{Q}, U+\bar{U}$, and $D+\bar{D}$. Both cases correspond to $\Delta \beta_{3}=4, \Delta \beta_{2}=3$, and $\Delta \beta_{1}=11 / 5$. The case $\Delta \beta_{3}=5$ (and the same $\beta$-function differences) would yield unification in the non-perturbative domain, but is also conceivable. In any case, these choices yield $S U(2) \times U(1)_{Y}$ unification slightly above the reduced Planck scale of $2.4 \times 10^{18} \mathrm{GeV}$. The prediction for $\alpha_{s}\left(M_{Z}\right)$ is now below typical observed values but the discrepancy is in general neither much better nor much worse than in the MSSM. If it is found 
that higher order or threshold effects tend to raise the prediction for $\alpha_{s}$, these scenarios may be of potential interest, but I have not considered them in the analysis of this paper. In this context it is amusing to note that one would obtain a very good prediction for $\alpha_{s}$, as well as unification very close to the string scale, if these extra fields have intermediate scale masses near the geometric mean of the string and electroweak scales.

Even given these possible directions it is possible to draw some general conclusions from the kind of analysis introduced in this work. Having left the framework of $E_{6}$ inspired $U(1)^{\prime}$ models, anomaly cancellation tends to provide $U(1)$ groups which have charges to the ordinary fermions just as the $U(1)$ subgroups of $E_{6}$. The charges of some of the extra fields typically differ, sometimes forbidding fast proton decay mediating couplings. A more thorough phenomenological investigation will be presented elsewhere.

\section{Acknowledgement:}

It is a pleasure to thank Paul Langacker and Michael Plümacher for many fruitful discussions. This work was supported in part by U.S. Department of Energy Grant EY-76-02-3071.

\section{References}

[1] For reviews see, H.P. Nilles, Phys. Rep. 110, 1 (1984);

H.E. Haber and G.L. Kane, Phys. Rep. 117, 75 (1985).

[2] J.E. Kim and H.P. Nilles, Phys. Lett. 138B, 150 (1984).

[3] A. Font, L.E. Ibáñez, and F. Quevedo, Phys. Lett. 228B, 79 (1989).

[4] T. Banks and L. Dixon, Nucl. Phys. B307, 93 (1988).

[5] S. Weinberg, Phys. Rev. D26, 287 (1982);

N. Sakai and T. Yanagida, Nucl. Phys. B197, 533 (1982);

L.J. Hall and I. Hinchliffe, Phys. Lett. 112B, 351 (1982).

[6] For a review see, M. Cvetič and P. Langacker, $Z^{\prime}$ Physics and Supersymmetry, p. 312 in Perspectives in Supersymmetry, ed. G.L. Kane (World Scientific, Singapore, 1998), e-print hep-ph/9707451.

[7] S. Dimopoulos, S. Raby, and F. Wilczek, Phys. Rev. D24, 1681 (1981);

L.E. Ibáñez and G.G. Ross, Phys. Lett. 105B, 439 (1981);

U. Amaldi et al., Phys. Rev. D36, 1385 (1987);

J. Ellis, S. Kelley, and D.V. Nanopoulos, Phys. Lett. 249B, 441 (1990);

P. Langacker and M.-X. Luo, Phys. Rev. D44, 817 (1991);

U. Amaldi, W. de Boer, and H. Fürstenau, Phys. Lett. 260B, 447 (1991);

P. Langacker and N. Polonsky, Phys. Rev. D47, 4028 (1993) and ibid. D52, 3081 (1995);

P.H. Chankowski, Z. Płuciennik, and S. Pokorski, Nucl. Phys. B439, 23 (1995);

J. Bagger, K. Matchev, and D. Pierce, Phys. Lett. 348B, 443 (1995). 
[8] J. Erler, Implications of Precision Electroweak Measurements for the Standard Model Higgs Boson, p. 40 in the Proceedings of the 17th International Workshop on Weak Interactions and Neutrinos (WIN 99), Cape Town, South Africa, January 1999, hep-ph/9904235.

[9] J. Erler and P. Langacker, Electroweak Model and Constraints on New Physics, in Ref. [10].

[10] D.E. Groom et al., Eur. Phys. J. C15, 1 (2000).

[11] M. Carena, M. Quiros, and C.E.M. Wagner, Nucl. Phys. B461, 407 (1996); D.M. Pierce, J.A. Bagger, K. Matchev, and R.J. Zhang, Nucl. Phys. B491, 3 (1997);

H.E. Haber, R. Hempfling, and A.H. Hoang, Z. Phys. C75, 539 (1997);

M. Masip, R. Muñoz-Tapia, and A. Pomarol, Phys. Rev. D57, 5340 (1998);

S. Heinemeyer, W. Hollik, and G. Weiglein, Phys. Rev. D58, 091701 (1998), Phys. Lett. 440B, 296 (1998), and Eur. Phys. J. C9, 343 (1999);

R.J. Zhang, Phys. Lett. 447B, 89 (1999).

[12] J. Erler and D.M. Pierce, Nucl. Phys. B526, 53 (1998);

G.C. Cho, K. Hagiwara, C. Kao, and R. Szalapski, Constraints on the mSUGRA Parameter Space from Electroweak Precision Data, to appear in the Proceedings of Physics at Run II: Workshop on Supersymmetry / Higgs: Summary Meeting, Batavia, IL, November 1998, e-print hep-ph/9901351.

[13] J. Erler and P. Langacker, Phys. Rev. Lett. 84, 212 (2000).

[14] C.S. Wood, et al., Science 275, 1759 (1997);

S.C. Bennett and C.E. Wieman, Phys. Rev. Lett. 82, 2484 (1999).

[15] Implications of a $Z^{\prime}$ for $Q_{W}$ have been emphasized in the third Ref. [7] and by W.J. Marciano and J.L. Rosner, Phys. Rev. Lett. 65, 2963 (1990);

P. Langacker, Phys. Lett. 256B, 277 (1991);

K.T. Mahanthappa and P.K. Mohapatra, Phys. Rev. D43, 3093 (1991); and recently by R. Casalbuoni, S. De Curtis, D. Dominici, and R. Gatto, Phys. Lett. 460B, 135 (1999); and J.L. Rosner, Phys. Rev. D61, 016006 (2000).

[16] N.H. Edwards et al., Phys. Rev. Lett. 74, 2654 (1995);

P.A. Vetter et al., Phys. Rev. Lett. 74, 2658 (1995).

[17] The LEP Collaborations, ALEPH, DELPHI, L3, OPAL, the LEP Electroweak Working Group, and the SLD Heavy Flavour and Electroweak Groups, A Combination of Preliminary Electroweak Measurements and Constraints on the Standard Model, preprint CERNEP-2000-016.

[18] M. Peskin and T. Takeuchi, Phys. Rev. Lett. 65, 964 (1990).

[19] G. Altarelli and R. Barbieri, Phys. Lett. 253B, 161 (1990).

[20] J.L. Hewett and T.G. Rizzo, Phys. Rep. 183, 193 (1989). 
[21] D. Suematsu and Y. Yamagishi, Int. J. Mod. Phys. A10, 4521 (1995).

[22] M. Cvetič and P. Langacker, Phys. Rev. D54, 3570 (1996).

[23] L.E. Ibáñez and G.G. Ross, Nucl. Phys. B368, 3 (1992);

A.H. Chamseddine and H. Dreiner, Nucl. Phys. B447, 195 (1995).

[24] M. Cvetic, D.A. Demir, J.R. Espinosa, L. Everett, and P. Langacker, Phys. Rev. D56, 2861 (1997) and ibid. D58, 119905 (1998).

[25] M. Aoki and N. Oshimo, A Supersymmetric Model with an Extra U(1) Gauge Symmetry, e-print hep-ph/9907481.

[26] S. Wolfram, Phys. Lett. 82B, 65 (1979);

E. Nardi and E. Roulet, Phys. Lett. 245B, 105 (1990).

[27] P. F. Smith, J. R. Bennett, G. J. Homer, J. D. Lewin, H. E. Walford, and W. A. Smith, Nucl. Phys. B206, 333 (1982);

T. K. Hemmick et al., Phys. Rev. D41, 2074 (1990).

[28] For a recent review and references see,

A. Riotto and M. Trodden, Ann. Rev. Nucl. Part. Sci. 49, 35 (1999).

[29] D. J. Chung, E. W. Kolb and A. Riotto, Phys. Rev. D60, 063504 (1999).

[30] E. Ma, Phys. Rev. D36, 274 (1987);

K.S. Babu et al., Phys. Rev. D36, 878 (1987);

J.F. Gunion et al., Int. J. Mod. Phys. A2, 1199 (1987).

[31] P. Langacker, private communication.

[32] P. Langacker and M. Plümacher, Flavor Changing Effects in Theories with a Heavy Z' Boson with Family Non-Universal Couplings, e-print hep-ph/0001204.

[33] For a discussion see, R. Hempfling, Phys. Lett. 351B, 206 (1995).

[34] A.N. Schellekens, Phys. Lett. 237B, 363 (1990).

[35] J. Erler, Nucl. Phys. B475, 597 (1996).

[36] P. Langacker, Phys. Rev. D58, 093017 (1998).

[37] Super-Kamiokande Collaboration: Y. Fukuda et al., Phys. Rev. Lett. 81, 1562 (1998). 